\title{
Immediate Implantation into extraction socket, a case report
}

\author{
Dr. Swapnil Moghe ${ }^{1}$, Dr. Anjali Moghe ${ }^{2}$,Dr. Rakesh $\mathrm{Shah}^{3}$,Dr. Navin Shah ${ }^{4}$ Dr. \\ Ajay Pillai ${ }^{5}$, Dr. Saksham Nahar ${ }^{6}$, Dr. Neha Goyal ${ }^{7}$ \\ ${ }^{I}$ (Reader, Department Of Oral \& Maxillofacial Surgery, Peoples Dental Academy, Peoples University, Bhopal, \\ Madhya Pradesh, India.) \\ ${ }^{2}$ (Ex. Lecturer, Oral Medicine \& Radiology, Peoples Dental Academy, Peoples University, Bhopal, Madhya \\ Pradesh, India.) \\ ${ }^{3}$ (Professor, Department Of Oral \& Maxillofacial Surgery, K.M.Shah Dental College \& Hospital, Vadodara, \\ Gujarat, India) \\ ${ }^{4}$ (Professor, Department Of Oral \& Maxillofacial Surgery, K.M.Shah Dental College \& Hospital, Vadodara, \\ Gujarat, India) \\ ${ }^{5}$ (Reader, Department Of Oral \& Maxillofacial Surgery, Peoples Dental Academy, Bhopal, India.) \\ ${ }^{6}$ (Post graduate Student, Department Of Oral \& Maxillofacial Surgery, Peoples Dental Academy, Bhopal, \\ India.) \\ ${ }^{7}($ Consultant Dental Surgeon, Govt. District hospital, Mungeli.)
}

\section{Summary}

Dental implants can be placed in fresh sockets just after tooth extraction. These are called 'immediate implants'. The advantages of immediate implants are that treatment time can be shortened and that bone height might be maintained thus possibly improving the aesthetic results. The potential disadvantages as per the studies are an increased risk of infection and failures of the immediately placed implants. We report a case of 65 year old patient with root stump in relation to right mandibular molar, who came for replacement of the same.

\section{Key Words: Immediate Implant, Osseointegration, Fresh extraction socket}

\section{Background}

Today, immediate implant placement into fresh extraction sockets, first introduced by Schulte et al in the late $1970 \mathrm{~s}^{1}$ is considered to be a predictable procedure. A series of well-controlled studies by Schwartz, Arad et al, investigated the influence of immediate implantation survival rates and comprised of a large number of immediately placed implants over 7 years. These suggested that immediately placed implants are as predictable as those implants placed in completely healed extraction sites, and that immediate implantation does not carry additional morbidity ${ }^{2,3}$.There are, however, some important factors to be considered in order to ensure this predictability, as well as long-term acceptable esthetic and functional outcomes.

Available bone height should be confirmed by careful radiological examination. Ideally, $2-3 \mathrm{~mm}$ of healthy, good quality, dense bone apical to the socket should be present in order to ensure good primary stability. A computed tomography (CT) scan is a very helpful tool in assessing bone quality and density, measured in Hounsfield units. The extraction socket should be free from acute pathology. This can be confirmed by preoperative radiographs and the absence of acute symptoms associated with the tooth to be extracted. The extraction socket should be examined carefully following extraction, in order to ensure intact walls. This can be performed using a ball-ended periodontal probe. Detected bone defects may require either guided bone regeneration or a bone grafting procedure, and the patient should be warned about this possibility.

Some animal studies, with their obvious limitations, indicated that chronic well-contained asymptomatic lesions can be curetted before implants are immediately placed, provided that no bone defects are present, and that the presence of these lesions does not compromise healing or osseointegration of the immediately placed implants. ${ }^{4}$ Soft tissue contour and volume should be sufficient to ensure acceptable profile and gingival health of the completed implant-supported restoration. The site should be free from periodontal disease to ensure stability of the soft and hard tissue around the implant.

The crestal and papillary bone level proximal to the site of the implantation should be stable, as this in turn will be dictating the stability of the proximal papillary height around the definitive restoration. Different teeth exhibit different anatomy, and this fact makes some teeth better candidates than others for immediate implantation. The bulbous contour of canine teeth roots renders them more likely to exhibit some labial dehiscence. Lower central and lateral incisors have fine roots that may result in labial perforation.

A surgical stent, based on a diagnostic wax-up, is often used to assist with implant positioning. In the anterior maxillary region, the site is usually developed slightly in a more palatal direction to that of the extracted tooth in 
order to avoid cortical plate perforation and improve primary stability, as well as to obliterate most of the extraction socket.

A pilot drill is used to establish the centre of the osteotomy. This pilot osteotomy will be gradually enlarged to achieve the diameter of the implant to be placed. This can be performed by using irrigated drills, osteotomes, or bone spreaders. In the posterior maxilla, osteotomes are usually preferred in order to improve bone density and bone/implant contact. The use of osteotomes and bone spreaders will also offer gradual and controlled site enlargement, thus minimizing the risk of perforation, especially when the procedure is performed with no flap reflection, i.e., blindly. During site enlargement, the bone plates should be continuously inspected for perforation, and gentle finger pressure on the labial plates should be maintained. The desired implant should then be inserted using the appropriate inserting device.

Manual placement offers controlled torque, as well as the advantage of "feeling" primary stability as the implant is introduced. The desired depth of the inserted implant will be dictated by the required aesthetic profile based on the protocol of the system used. If immediate loading is planned, no closure is required. If however, there are doubts regarding primary stability, or if the occlusion is not favourable, then wound closure would be required. If primary closure cannot be achieved-as in the case of wide sockets and limited soft tissue, occluding membranes or soft tissue grafts can be considered. Placement of dental implants into fresh extraction sockets offers a number of significant advantages. Without the support provided by functioning teeth or implants, extraction sockets undergo a catabolic phase as a part of the remodelling process. This results in varying amounts of atrophy after tooth removal. Recession of the mucosal and gingival tissues accompanies bone remodelling. As a result, achievement of superior aesthetics becomes more difficult, particularly in the aesthetic zone. Immediate placement offers minimal changes to the healing bone and soft tissues, leading to better aesthetic and functional outcomes.

Furthermore, implants in fresh extraction sockets can be placed in almost the same position as the extracted tooth, facilitating the positioning of the final restoration within the same prosthetic envelope without the need for severe angulation of the abutment or fabrication of telescopic copings. This results in superior aesthetic and functional outcomes. Finally, eliminating the standard wait for primary healing of the soft and hard tissues significantly shortens the overall treatment time and the interval during which the patient must live in a transitional state with or without teeth. The reduction of the number of surgical appointments and associated surgical trauma, as well as less overall treatment costs, increases patient's acceptance of implant treatment. ${ }^{8}$

Immediate implant placement requires some strict parameters for success and should be decided upon following a careful planning stage.

The ideal situation for immediate implant placement is where the infection-free, intact socket can be obliterated almost entirely by the implant. With increasing gap size between the implant and the socket, the amount of bone-to-implant contact is reduced. Situations where single teeth need to be replaced by means of an immediate implant depend on the adjacent teeth to sustain the level of bone. In situations where multiple teeth need to be extracted and immediately replaced, some concerns regarding the ability to sustain bone levels may be raised. Loss of bone between the implants may result in loss of interdental papillae. This may result in having to embark on adjunctive hard tissue/soft tissue grafting/augmentation procedures that defy the object of performing immediate implant placement in the first place.

Anatomical variations and different root patterns of different teeth can result in some difficulties with immediate implantation. The limited vertical height caused by the presence of the maxillary sinus in the posterior maxilla and inferior dental nerve in the posterior mandible, coupled with the presence of wide sockets, may tempt the clinician to use larger diameter but shorter implants, thus relying on engaging the socket walls in order to achieve sufficient primary stability. This would, in turn, carry the risk of perforation or placing implants outside the pre-planned prosthetic envelope.

\section{Case Presentation}

A 65 year old male patient reported in Postgraduate Department of Oral \& Maxillofacial Surgery \& Oral Implantology, KMSDCH, Vadodara. Patient complained of inability to eat with lower Right back tooth region since 1 year. On examination, the teeth were deemed unrestorable (Fig. 1) \& patient insisted on having implants .Our only concern was that patient was medically compromised having undergone CABG 2 years back along with Diabetes \& Hypertension. After thorough clinical \& radiographic examination, we decided to go for immediate implantation i.r.t. 46, 47 under proper surgical asepsis \& a standard protocol was followed. Two grams of amoxicillin was prescribed to be taken 1 hour before surgery. Local anaesthesia was achieved \& atraumatic extraction of $46 \& 47$ was done with use of periotomes.

When appropriate depth was reached with the pilot drill, the implant depth probe was used for tactile perception of intact bony plates $\&$ or any perforations $\&$ the confirmation of desired osteotomy depth. Once desired depth was confirmed, paralleling pins were placed (Fig. 2) to check the proper alignment of the implant with adjacent teeth $\&$ opposing occlusion. After confirmation of depth $\&$ angulation of the osteotomy site, The 
implant was removed from the sterile vial using the insertion tool and delivered directly into the osteotomy site. Contamination by touching the implant with instruments made of a dissimilar metal or by contact with soft tissue, cloth or even surgical gloves may affect the degree of osseointegration.

The implant was then pressed into the prepared site with manual pressure aided by the insertion mount \& insertion tool attached to the implant head \& a $4.8 \mathrm{~mm}$ wide \& $10 \mathrm{~mm}$ long CSM implant was placed. the socket was carefully modified with appropriate implant drills. Following which, the insertion mount was removed and hex driver was placed into the implant internal hex \& ratcheted with torque-controlled implant ratchet. Implant was never forced into the socket with excessive force as this might lead to micro cracks in the, surface bone resulting in improper osseointegration. Implant that was placed was checked for stability by applying gentle pressure to determine if it could be depressed or rotated. Also, primary implant stability was assessed with the torque controlled ratchet.

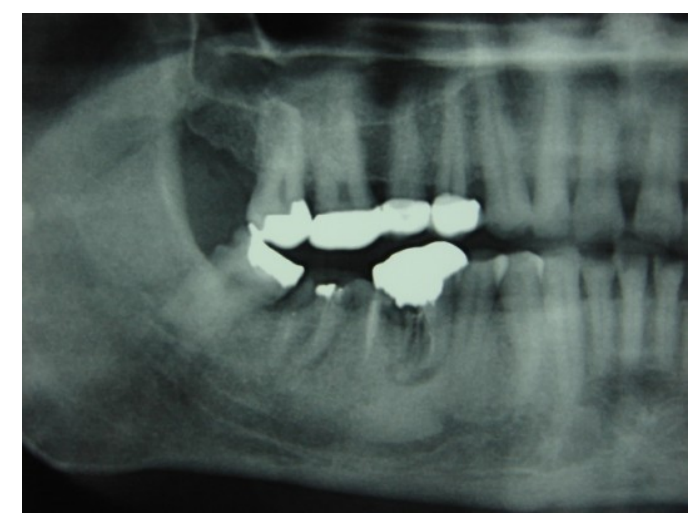

Fig 1: Preoperative OPG Showing Unrestorable 46, 47 teeth.

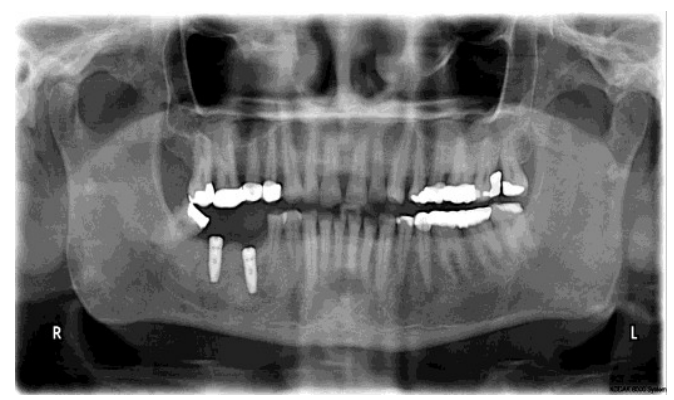

Figure 3: Immediate Post Operative OPG after Implant Placement.

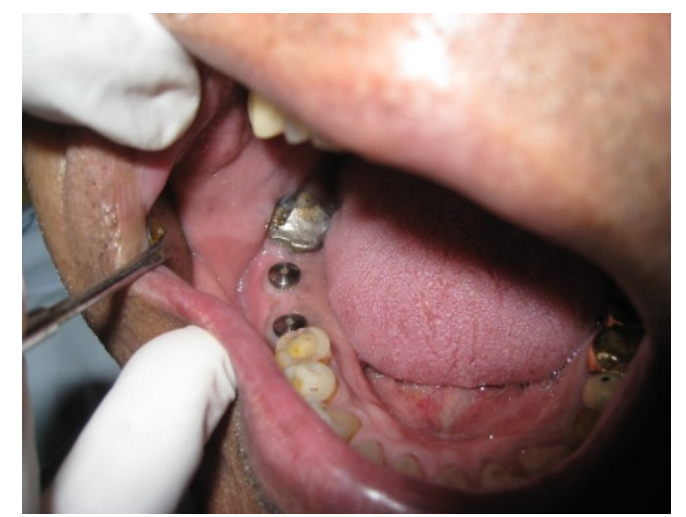

Fig. 5: Gingival former in place w.r.t 46, 47 .

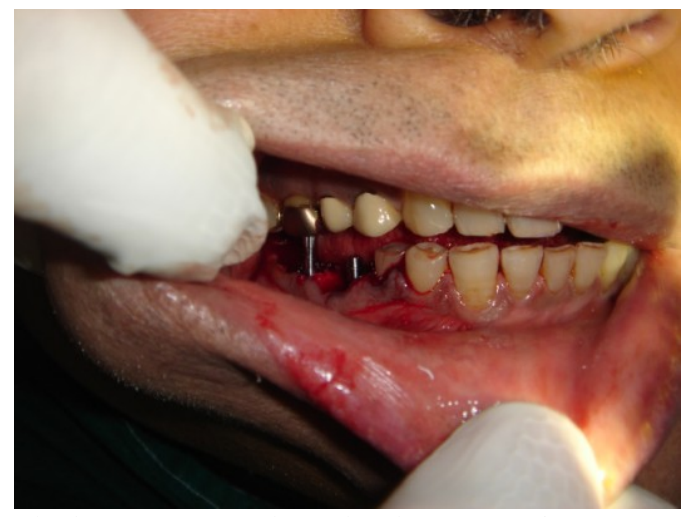

Fig.2: Implant guide pins in place after atraumatic extraction of tooth.

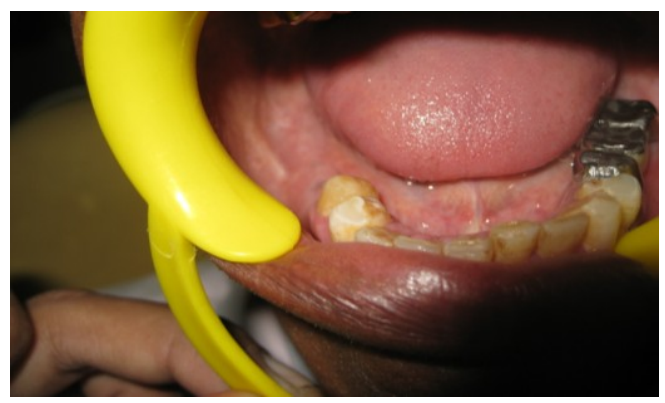

Fig. 4: Postoperative healing after 3 months of Implant placement.

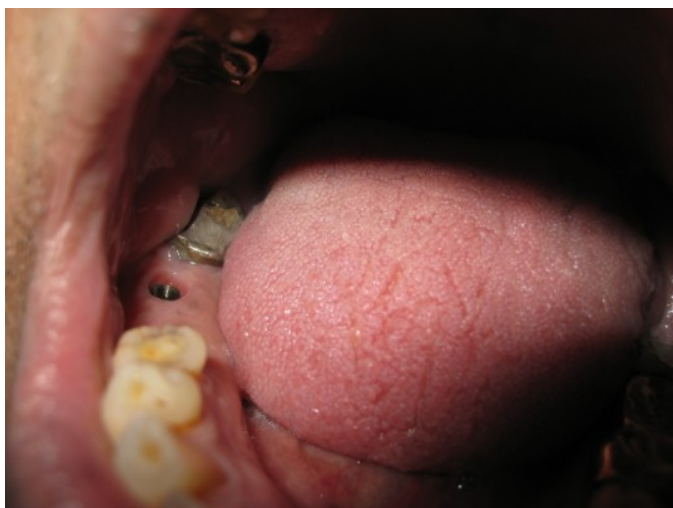

Fig. 6: Gingival collar formed after removal of gingival seal. 


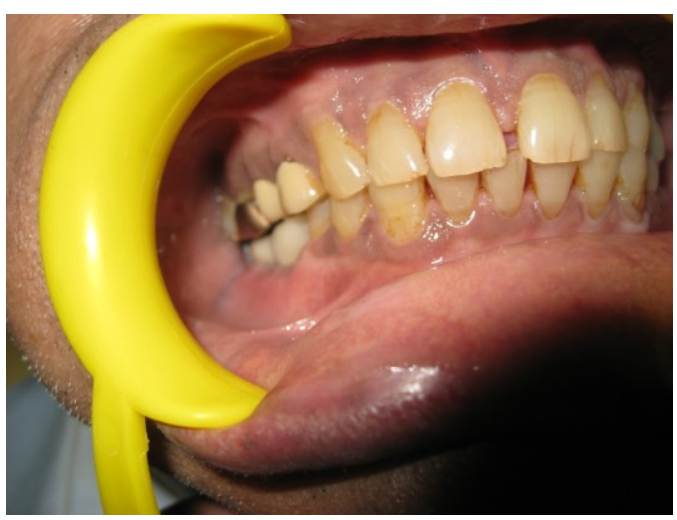

Fig. 7: P.F.M Crown in place w.r.t 46, 47.

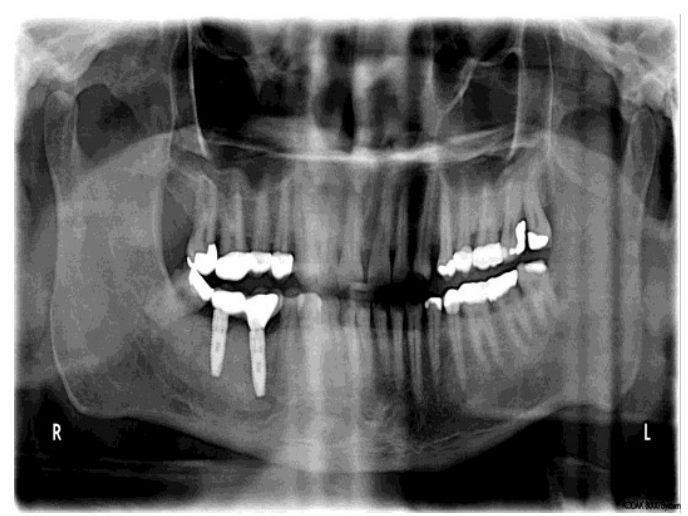

Fig.8: One year Postoperative OPG after placement of P.F.M Crown w.r.t. 46, 47.

The cover screw, provided with the implant package was then placed using the hex-driver using finger pressure. At this point, implant was confirmed to be immobile, which re-affirmed primary implant stability. The buccal surface \& marginal voids were grafted with Bio Oss \& torque was in excess of $30 \mathrm{~N}-\mathrm{cm}$. Primary closure was achieved with black silk 3-0.Amoxycillin $500 \mathrm{mg}$ TDS was prescribed for 1 week, and chlorhexidine mouthwash was prescribed for 3 weeks. Subsequent healing was uneventful. A radiograph was taken post operatively (Fig. 3) to evaluate the implant angulation \& position. The patient was on regular recall and under strict oral hygiene measures. Healing was good as evident in Figure 4. Stage 2 surgery to uncover the implant was performed after a period of 3 months \& gingival former were placed (Fig.5). During surgery, all the threads on buccal surface were found to be covered with bone. Radiograph was taken and prosthetic phase was carried out under the opinion \& supervision of a prosthodontist. After soft tissue healing, gingival seal was evident (Fig. 6), Impressions were made with the impression post attached to the implant using the open tray impression technique. Shade selection was also done during this appointment. Casts with impression postimplant analogue complex, abutment, lab drivers and selected shade were sent to laboratory for preparation of cement retained porcelain fused to metal crown. Healing abutment/ gingival former was replaced till the time taken for laboratory manufacture of prosthesis. After approx. 4-7 days, the healing abutments were removed and replaced with final abutment. The PFM crown was checked for its passive fitting to abutment and noninterference with adjacent teeth. Crown was then cemented with glass-ionomer cement (Fig.7). The patient was dispatched with a reminder of oral hygiene instructions \& the recall programme. A postoperative OPG was taken after 1 year of Implant placement, which was suggestive of good Osseo integration i.r.t. implant site (Figure 8).

\section{Investigations \\ 1. Orthopantomogram \\ 2. IOPA \\ 3. Complete blood count (CBC)}

\section{Discussion}

A consensus committee report prepared by Ganeles and coworkers reviewed the available literature published predominantly in refereed journals and concluded that most of the reviewed studies demonstrated lower success rate with machined-surface implants, particularly in poor-quality bone ${ }^{5}$.Implants with a sparse thread pattern also have been found to increase success rates of immediately placed implants. Few investigators have directly compared the integration rates of roughened, threaded surfaces with those of machined surfaces. Glauser and coworkers noted a significant increase in success rate when using threaded implants with rough surfaces compared to machine-surface implants, and the difference was particularly striking when evaluating both types of implants in poor-quality bone. ${ }^{6}$

The importance of clinical and radiological examinations of the tooth to be extracted cannot be overstressed. These offer valuable information regarding potential difficulties, such as unfavourable root patterns and the presence of pathology. Extraction should be performed atraumatically, using periotomes and fine-peaked elevators specially designed for this purpose. Difficult teeth or roots can be sectioned by high-speed drills \& elevated separately. A gentle finger pressure on the buccal plate during elevation can minimize microfractures of the bony plate. Where possible, full-thickness mucoperiosteal flaps should be avoided in order to maintain blood supply to the healing socket. Following extraction, the socket should be irrigated with sterile saline and thoroughly inspected for fragments or defects. 
Many clinical reports and experimental studies in the animal model demonstrated the favourable outcome of dental implants immediately inserted in freshly extraction socket, without the use of any regenerative materials ${ }^{9,10}$. The outcome is always better when regenerative materials like Bio-Oss \& PRP are used $^{11}$.Our data shows a survival rate of immediate implantation carried out on this patient at three year after immediate implantation and does not differ from the cases in which implant was placed in healed sites. These data agree with those from other authors who evaluated the clinical success rate of immediate implantation without use of any membrane or graft material in both humans and animals. It must be kept in mind that the present study is related to immediate implant not subjected to functional loading and therefore not fully comparable with the results from loaded implants. However, it has been demonstrated that functional loading does not impair, but rather enhances, bone maturation.

\section{Conclusion}

Immediate implant placement results in the preservation of bone height $\&$ width $\&$ is a useful tool to rehabilitate the patient.

\section{Acknowledgment}

The authors would like to thank Dr Rakesh Shah, Dr. Anjali Moghe \& Dr. Kiran Desai for their constant encouragement \& support. Without them this work would be unfinished.

\section{Learning Points/Take Home Messages}

1. Immediate implant placement avoids the unnecessary long recall appointments \& waiting period associated with the delayed implant placement.

2. Immediate implant placement preserves the bone height $\&$ width, which is lost due to bone resorption in case of Delayed Implant placement.

3. Implants in fresh extraction sockets can be placed in almost the same position as the extracted tooth, facilitating the positioning of the final restoration within the same prosthetic envelope without the need for severe angulation of the abutment or fabrication of telescopic copings. This results in superior esthetic and functional outcomes.

\section{References}

[1]. Wilson G, Schenk R, Buser D, et al (1998) Implants placed in immediate extraction sites: a report of histologic and histometric analysis of human biopsies. Int J Oral Maxillofac Implants13: 333-341.

[2]. Schwartz-Arad D, Caushu G (1997) The ways and where fores of immediate placement of implants into fresh extraction sites: a literature review. J Periodontol68: 915-923.

[3]. Schwartz-Arad D, Caushu G (1997) Placement of implants into fresh extraction sites: 4-7 years retrospective evaluation of 95 immediate implants. J Periodontol68:1110-1116.

[4]. Novaes AB Jr, Vidigal GM Jr, Novaes AB, et al (1998) Immediate implants placed into infected sites. a histomorphometric study in dogs. Int J Oral Maxillofac Implants13:422-427.

[5]. Ganeles J, Wismeijer D (2004) Early and immediately restored and loaded dental implants for single tooth and partial arch applications. Int J Oral Maxillofac Implants19 (Suppl):92-102.

[6]. Glauser R, Lundgren AK, Gottlow J, et al (2003) Immediate occlusal loading of Branemark Ti Unite implants placed predominantly in soft bone: 1-year results of a prospective clinical study. Clin Implant Dent Relat Res, 5(Suppl 1):47-55.

[7]. Sethi A, Kaus T (2005). Practical Implant Dentistry: diagnostic, surgical, restorative, and technical aspects of aesthetic and functional harmony. Quintessence Co. Ltd: 75-79.

[8]. Gomez-Roman G, Kruppenbacher M, Weber H (2001) Immediate post extraction implant placement with root-analog stepped implants: surgical procedure and statistical outcome after 6 years. Int J Oral Maxillofac Implants 16:503-513.

[9]. Fugazzotto PA. Treatment options following single-rooted tooth removal: A literature review and proposed hierarchy of treatment selection. Journal of Periodontology 2005; 76(5):821-31.

[10]. Yukna RA. Clinical comparison of hydroxyapatite-coated titanium dental implants placed in fresh extraction sockets and healed sites. Journal of Periodontology 1991; 62(7):468-72.

[11]. Moghe S etal, Platelet-rich Plasma in the Periodontal defect treatment after extraction of impacted third molar, NJMS/Vol.III/ Issue II) July 2012 